\title{
Thermal-Sensing Fiber Devices by Multimaterial Codrawing**
}

\author{
By Mehmet Bayindir,* Ayman F. Abouraddy, Jerimy Arnold, John D. Joannopoulos, and Yoel Fink*
}

Thermal sensing and thermography yield important information about the dynamics of many physical, chemical, and biological phenomena. ${ }^{[1,2]}$ Spatially resolved thermal sensing enables failure detection in technological systems when the failure mechanism is correlated with localized changes in temperature. Indeed, IR-imaging systems have become ubiquitous for applications where line-of-sight contact can be made between the measured object and the camera lens. Nevertheless, many critical applications do not lend themselves to radiative IR imaging because of the subterraneous nature of the monitored surface, spatial constraints, or cost considerations. The recent challenge of monitoring the skin temperature beneath the thermal tiles on the space shuttle represents a good example in which high-spatial-resolution information is required on very large surface areas but which cannot be obtained using traditional thermal-imaging systems. Thus, the problem of continuously monitoring and detecting a thermal excitation on very large areas $\left(100 \mathrm{~m}^{2}\right)$ with high resolution $\left(1 \mathrm{~cm}^{2}\right)$ is one that has remained largely unsolved. ${ }^{[3,4]}$ We present a new methodology for measuring spatially resolved temperature information on large areas with high spatial resolution and low cost. Underlying our approach is a new fiber material that senses heat along its entire length and generates an electrical signal. This is in contrast to all previous work on thermal sensing using fibers, which require the use of optical probing signals. ${ }^{[5]}$

Although the fibers are produced by thermal drawing, they contain a set of materials that have not been traditionally associated with this process. The use of thermal drawing guaran-

[*] Prof. M. Bayindir, Prof. Y. Fink, Dr. A. F. Abouraddy, J. Arnold, Prof. J. D. Joannopoulos

Research Laboratory of Electronics

Massachusetts Institute of Technology

Cambridge, MA 02139 (USA)

E-mail:mehmet@mit.edu; yoel@mit.edu

Prof. M. Bayindir

Department of Physics, Bilkent University

06800 Bilkent, Ankara (Turkey)

Prof. Y. Fink

Department of Materials Science and Engineering

Massachusetts Institute of Technology

Cambridge, MA 02139 (USA)

Prof. J. D. Joannopoulos

Department of Physics, Massachusetts Institute of Technology Cambridge, MA 02139 (USA)

[**] This work was supported by the U.S. Army through the Institute for Soldier Nanotechnologies, under Contract DAAD-19-02D0002, DARPA DAAD19-03-1-0357, DOE DE-FG02-99ER45778. This work was also supported in part by the MRSEC Program of the National Science Foundation under award number DMR 02-13282. tees the production of extremely long fibers, while the innovation in preparation of the preform and choice of materials allows the incorporation of novel functionalities. Specifically, both thermal and electrical functionalities are obtained in the fibers studied in this communication, while optical and optoelectronic functionalities in alternative designs have been obtained previously and are reported elsewhere. ${ }^{[6,7]}$

The fibers are produced by a novel fabrication technique that enables the incorporation of materials with widely disparate electrical and thermal properties in a single, macroscopic, cylindrical preform rod, which subsequently undergoes thermal drawing to give solid-state microstructured fibers with high uniformity. The main requirements in the materials used in this preform-to-fiber approach are as follows: 1) the component which supports the draw stress should be glassy, so as to be drawn at reasonable speeds in a furnace, with self-maintaining structural regularity; 2) the materials must be above their respective softening or melting points at the draw temerature to enable fiber codrawing; and 3) the materials should exhibit good adhesion/wetting in the viscous and solid states without delamination, even when subjected to thermal quenching. According to these requirements, we identified suitable semiconducting, insulating, and metallic materials. The insulating material is a $75 \mu \mathrm{m}$ thick polymer film: polysulfone (Ajedium, USA), having a glass-transition temperature, $T_{\mathrm{g}}=190^{\circ} \mathrm{C}$. The chosen semiconducting glass, $\mathrm{Ge}_{17} \mathrm{As}_{23} \mathrm{Se}_{14} \mathrm{Te}_{46}$ (GAST), was arrived at by optimizing the composition formula $\mathrm{Ge}_{x} \mathrm{As}_{40-x} \mathrm{Se}_{y} \mathrm{Te}_{60-y} \quad(10<x<20$ and $10<y<15)$ under constraints of compatibility of $T_{\mathrm{g}}$ and viscosity with the codrawn polymer. Metallic electrodes are made of the alloy $96 \% \mathrm{Sn}-4 \% \mathrm{Ag}$, which has a low meltingtemperature range $\left(T_{\mathrm{M}}=221-229^{\circ} \mathrm{C}\right)$ below the fiber-drawing temperature of $270^{\circ} \mathrm{C}$. The chemical composition of the glass is chosen such that the electronic mobility gap of the amorphous semiconductor is small, ${ }^{[8]}$ yielding high electrical responsivity to small changes in temperature. ${ }^{[7]}$

The fabrication process (see Fig. 1A) begins with preparing cylindrical rods of the glass (see "Amorphous Semiconductor Synthesis" in the Experimental section). A cylindrical shell of polymer having an inner diameter equal to that of the glass rod is prepared with four slits removed from the walls. Four thin rods of the metal alloy are then placed in these slits. The glass rod is inserted into the polymer shell (Fig. 1A(a)), and a polymer sheet is then rolled around the resulting cylinder to provide a protective cladding (Fig. 1A(b)). Finally, the cylinder is thermally consolidated (Fig. 1A(c)) and subsequently is drawn in a fiber-draw tower producing hundreds of meters of 
A

(a)

(b)

(c)
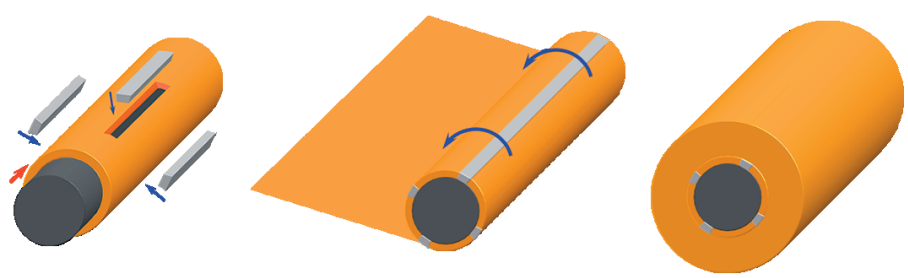

Insulator

Semiconducto

Metal

B

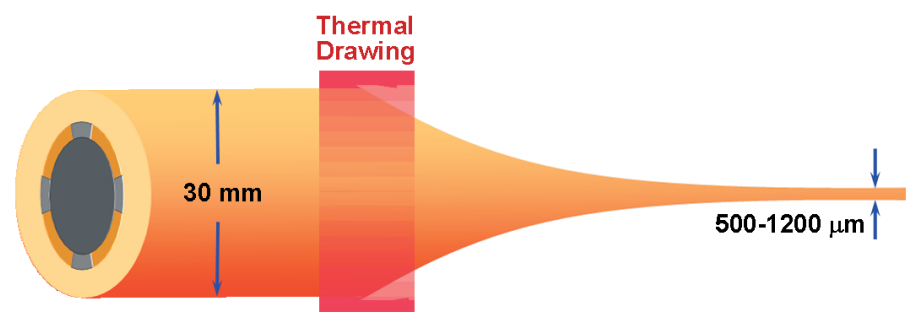

C (a)

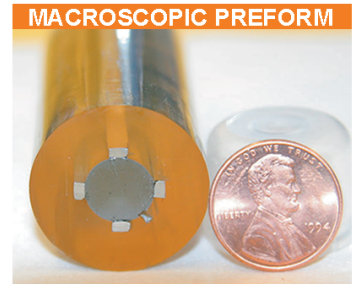

(b)

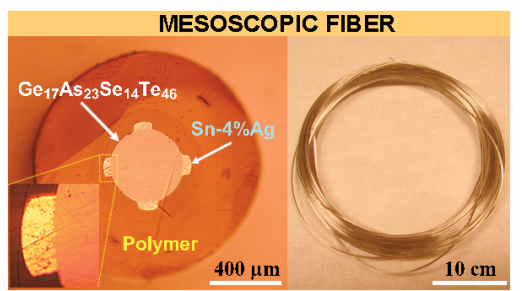

Figure 1. A) Fabrication steps for the fiber perform: a) a chalcogenide semiconducting glass rod is assembled with an insulating polymer shell and four metal electrodes; b) a polymer sheet is rolled around the structure to form a protective cladding; and c) the preform is consolidated in a vacuum oven. B) The preform is thermally drawn to mesoscale fibers. C) Images of the preform and fiber cross section: a) photograph of the fiber preform rod; b) a micrograph of a thermal-sensing fiber cross section (outer diameter is $1150 \mu \mathrm{m}$ ). The inset shows the quality of the metal/semiconductor interface; and c) a photograph of a $10 \mathrm{~m}$ long, flexible-fiber-based heat-sensing device.

fiber (Fig. 1B) (see "Preform Preparation and Fiber Drawing" in the Experimental section). The drawn fiber maintains the geometry and structure of the macroscopic preform (Fig. 1C(a)) even though molten metals have very small viscosities; this is facilitated by the confinement of the molten metals between high-viscosity semiconducting and insulating interfaces. Intimate contacts are formed at the glass/metal interfaces, as shown in Figure $1 \mathrm{C}(\mathrm{b})$. The fibers are flexible, lightweight, and protected (electrically and chemically) from environmental effects (Fig. 1C(c)). When the metal electrodes are connected to an external circuit, a functional thermalsensing device is obtained.

We characterized the thermal response of the chalcogenide glass in two distinct forms. In the first, a bulk sample is prepared by cutting a disk $(6.5 \mathrm{~mm}$ in diameter, $1.3 \mathrm{~mm}$ thick $)$ from the chalcogenide glass rod used in the preform and painting both sides with silver paint for electrical contact after polishing the two end surfaces. Measurements on this sample provide us with the intrinsic glass properties. The second sample is a section of the drawn fiber $(1150 \mu \mathrm{m}$ outer diameter, $9 \mathrm{~cm}$ long) with electrodes contacted using the same silver paint, providing us with the properties of the glass incorporated into a fiber device after thermal drawing (Fig. 2A(a)). Both samples were placed inside a pyrex tube surrounded by an electrical resistive heater, and the temperature was measured by means of a thermocouple (K-type) placed inside the tube alongside the two samples. Measurements below room temperature were carried out by placing the samples and the thermocouple in cold water. The electrical resistances of the two samples, as a function of temperature are plotted in Figure 2A(b) (measured using a Keithley 2000 multimeter). It is clear from these curves that the resistances of the two samples are best described with an exponential relation of the form $R \propto \exp \left(\Delta E / k_{\mathrm{B}} T\right),{ }^{[9-11]}$ where $k_{\mathrm{B}}$ is the Boltzmann constant, $T$ is the absolute temperature, and $\Delta E$ is the thermal activation energy. This behavior is maintained over almost four orders of magnitude of the resistance values. Measurements of the bulk sample yield a room-temperature resistivity of $2.3 \times 10^{6} \Omega \mathrm{cm}$ for the GAST glass. The mobility gap for amorphous glasses is typically twice $\Delta E .^{[10,11]}$ The measurements yield a value of $\Delta E=0.58 \mathrm{eV}$ for both samples, which indicates that the activation energy of the chalcogenide glass has not changed after thermal drawing. This value of $\Delta E$ is consistent with previously reported measurements for similar compositions of GAST glasses: $\mathrm{Ge}_{15} \mathrm{As}_{35} \mathrm{Se}_{10} \mathrm{Te}_{40}(\Delta E=0.45 \mathrm{eV})^{[8]}$ and $\mathrm{Ge}_{15} \mathrm{As}_{25^{-}}$ $\mathrm{Se}_{15} \mathrm{Te}_{45}(\Delta E=0.5 \mathrm{eV}) .{ }^{[7]}$

After characterization of the thermal response of the glass in bulk and fiber forms, we next studied the electrical response of the fibers. To this end we obtained the current-voltage $(I-V)$ curves for the fiber sample below and above room temperature $\left(11\right.$ and $58^{\circ} \mathrm{C}$, respectively). The measurement results (plotted in Fig. 2B(a)) clearly indicate that the device is ohmic over the studied temperature range. We have also examined the temporal response of the fiber sample after heating above, and cooling below, room temperature (Fig. 2B(b)). The fiber was heated by dipping it in hot water and was cooled by exposure to liquid nitrogen. The relaxation of the fiber resistance to its equilibrium value at room temperature after removal of the thermal excitation is fitted to an exponential curve.

Each fiber produces an electrical signal that is proportional to the integral of the thermal excitation along its whole length and thus cannot provide spatially resolved thermal measurements. Nevertheless, spatially resolved information may be ob- 
A

(a)

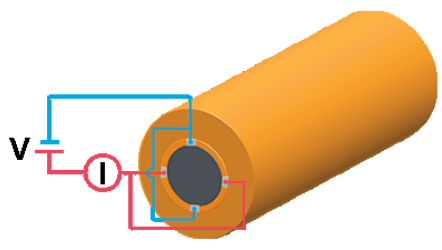

B

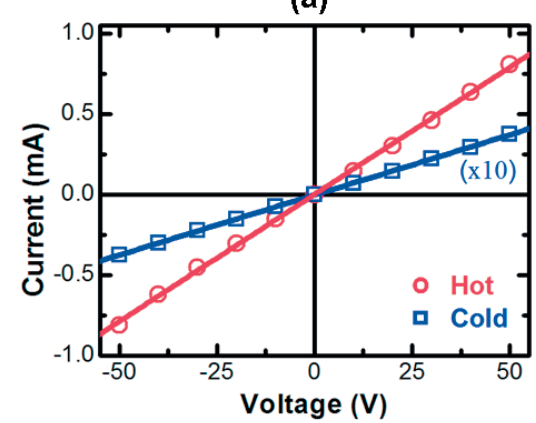

(b)

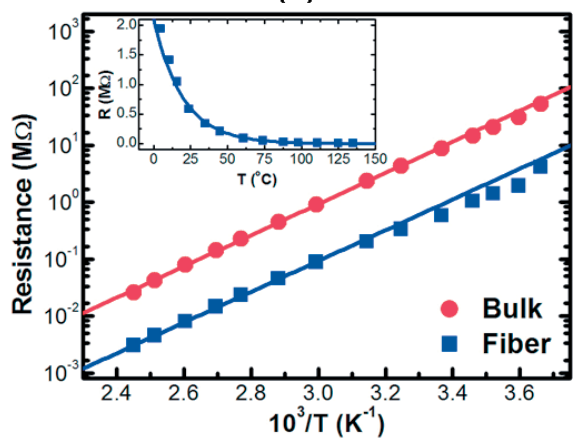

(b)

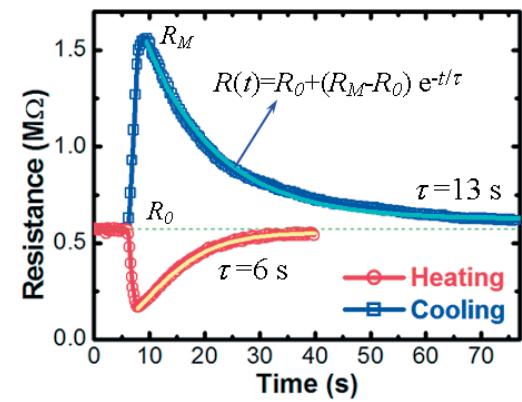

Figure 2. Thermal and electrical properties of the metal/semiconductor/metal (MSM) heat-sensing fiber device. A) A fiber device connected to a circuit through metallic electrodes is shown in (a). Resistance of a $9 \mathrm{~cm}$ long fiber and a bulk sample of the same glass as in the fiber, as a function of temperature, is given in (b). The inset shows the resistance of the fiber sample on a linear scale. B) The current-voltage (I-V) curve of the fiber MSM device at two different operating temperatures (Hot: $58^{\circ} \mathrm{C}$, Cold: $11^{\circ} \mathrm{C}$ ) is given in (a). The typical temporal response of the MSM device after heating and cooling the device fiber is shown in (b). ( $\tau$ : relaxation lifetime; $R_{0}$ : room-temperature resistance; $R_{\mathrm{M}}$ : maximum resistance.)

tained by assembling the fibers into an array, which serves the purpose of localizing the thermal excitation. An example of such an array is given in Figure 3; the fibers are arranged on a grid structure while embedded in a fabric, forming an $8 \times 8$ array with $1 \mathrm{~cm}$ separation distance between neighboring fibers. Moreover, the flexibility of the fibers allows them to conform easily to curved surfaces, such as the mannequin head shown in Figure 3. Each fiber is connected to an external circuit via the metal electrodes, and a signal proportional to the fiber resistance (and, thus, inversely proportional to temperature) is digitized in real time. A thermal map is reconstructed using these signals, as explained in the Experimental section.

We present reconstructed differential thermal maps in two situations: A) localized heating by the touch of a finger, and B) cooling with an ice cube. In each of these cases, we show a thermal IR image taken with an IR camera (FLIR, ThermaCam S60) and a reconstructed thermal map using the data obtained from the fiber array. These thermal maps are corroborated by referencing them to the thermal images obtained by the thermal IR camera and calibrating the fiber-array response accordingly. These results confirm the capability of localizing the source of thermal excitation to within the resolution of the fiber placement in the array.

Since the electrical signals from the fibers are acquired in real time, dynamical thermal maps may thus be obtained. In Figure 4, we present an example of a dynamical thermal-map acquisition using a fiber array. An array at room temperature is heated momentarily using a heat gun (corresponding, on the temporal axis, to the red bar). We monitor the temperature of the fabric using the IR camera while simultaneously processing the data obtained from the array. The panels in Figure 4 show synchronized thermal maps obtained using these two different methods, and good agreement is observed when using the above described calibration. The temperature resolution of the IR camera is specified to be $0.08^{\circ} \mathrm{C}$ at $30^{\circ} \mathrm{C}$. We obtained an estimate for the corresponding value for our fibers to be $0.1^{\circ} \mathrm{C}$ at $30^{\circ} \mathrm{C}$. We obtained this value by measuring the maximum fluctuation in the resistance of a fiber maintained at a specified temperature for several minutes.

The cooling, as seen in Figure 4, was slow because the fabric retained heat, as seen in the IR camera images. Several strategies are available to achieve a faster response time. In one approach, the solid glass core is replaced with a thin glass film, ${ }^{[7]}$ resulting in a faster response time. Further improvement is attained by reducing the fiber diameter. We expect that the poor thermal conductivity of the polymer $\left(\kappa=0.26 \mathrm{~W} \mathrm{~m}^{-1} \mathrm{~K}\right.$ for polysulfone) contributes to the slow decay time in the thermal response. Thus, a second strategy is to place additional metallic elements $\left(\kappa=66 \mathrm{~W} \mathrm{~m}^{-1} \mathrm{~K}^{-1}\right.$ for $\left.\mathrm{Sn}\right)$ in contact with the thermal sensing film on the inside (away from the source of heat) to act as a heat sink. 
A
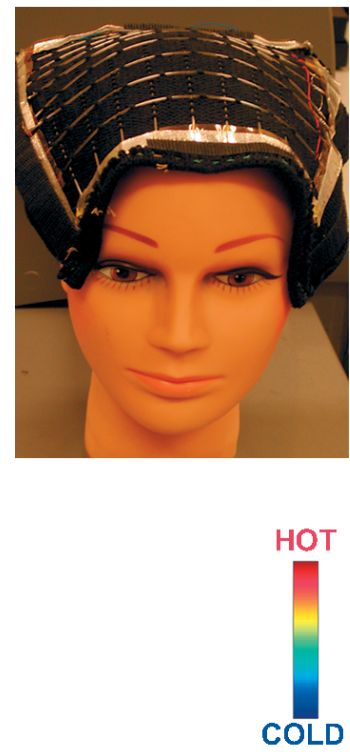
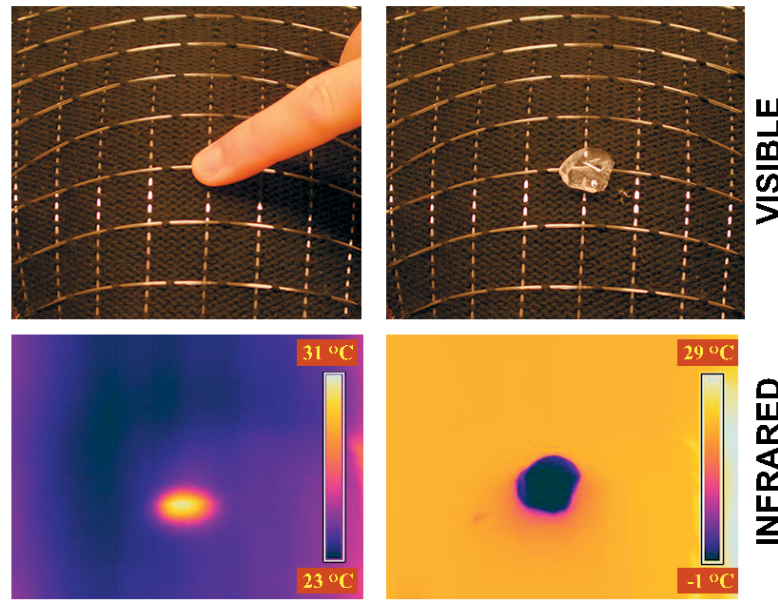

$\frac{w}{\frac{m}{9}}$
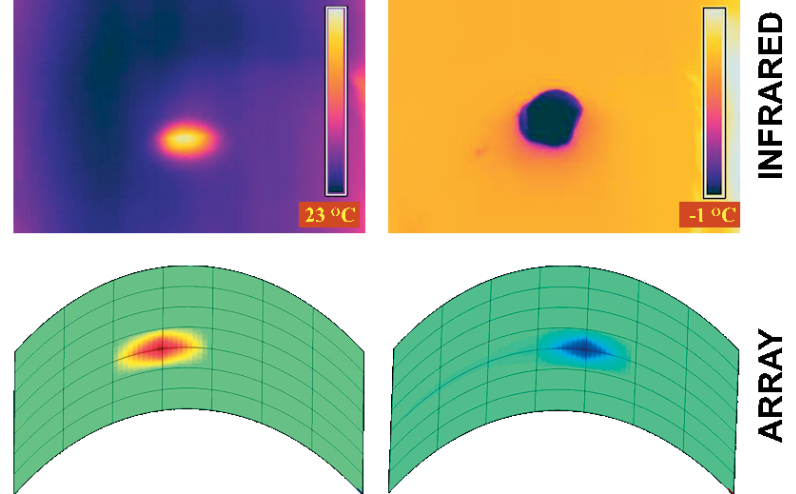

Figure 3. Temperature mapping with mesoscopic-fiber fabrics. The flexibility of an array constructed from thermal-sensing fibers is shown by placing it on a mannequin head. Results are presented for two cases: a) heating due to the touch of a finger; and b) cooling with an ice cube. The first row shows photographs of the two cases, the second row shows thermal IR-camera images obtained of the head after removing the source of excitation, and the third row shows the thermal maps reconstructed from the fiber data taken simultaneously with the IR camera. The array is an $8 \times 8 \mathrm{grid}$ with $1 \mathrm{~cm}$ resolution.

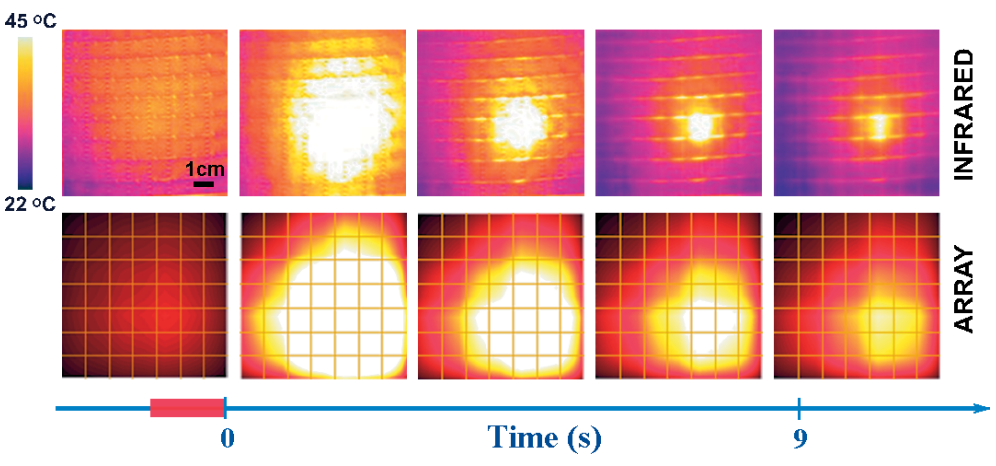

Figure 4. Dynamical thermal-map acquisition. The first row shows frames from a movie, acquired by a thermal IR camera, of a fiber array heated momentarily with a heat gun (corresponding to the red rectangle on the temporal axis). The second row shows reconstructed thermal maps (acquired in real time from the array data) at corresponding instances of time.

In summary, we have presented a process that involves the thermal codrawing of a macroscopic preform containing conductors, semiconductors, and insulators into very long fiber thermistors. The electrical conductivity of the fiber is modified by heat creating an electrical signal that is delivered to the fiber ends. The fibers are lightweight and flexible and may, consequently, be incorporated into fabrics or any other host structure. This work paves the way to large-contact-area temperature sensing at high spatial resolution.

\section{Experimental}

Amorphous Semiconductor Synthesis: The chalcogenide glass rod $\left(\mathrm{Ge}_{17} \mathrm{As}_{23} \mathrm{Se}_{14} \mathrm{Te}_{46}\right), 10 \mathrm{~mm}$ in diameter and $15 \mathrm{~cm}$ long, was prepared from high-purity $(5-6 \mathrm{~N}) \mathrm{Ge}$, As, Se, and Te elements (Alfa Aesar) using conventional sealed-ampoule melt-quenching techniques [12]. The materials were weighted and placed into a quartz tube under a nitrogen atmosphere. The tube was heated to $330^{\circ} \mathrm{C}$ for an hour at a rate of $1^{\circ} \mathrm{C} \mathrm{min}^{-1}$ under vacuum in order to remove surface oxides. The ampoule was formed by sealing the tube under vacuum $\left(\sim 10^{-5}\right.$ torr; $\left.\approx 1.33 \mathrm{mPa}\right)$. It was then heated to $950{ }^{\circ} \mathrm{C}$ at a rate of $2{ }^{\circ} \mathrm{C} \mathrm{min}{ }^{-1}$ in a rocking furnace for $18 \mathrm{~h}$, while being held vertically, and then rocked for $6 \mathrm{~h}$ to increase mixing and homogenization. The glass liquid was cooled to $710^{\circ} \mathrm{C}$ in the rocking furnace and then quenched in cold water. Subsequently, it was annealed for $30 \mathrm{~min}$ near the glass-transition temperature, $T_{\mathrm{g}} \sim 190^{\circ} \mathrm{C}$, before being cooled gradually to room temperature.

Preform Preparation and Fiber Drawing: The hundreds of meters of fiber were obtained from a macroscopic cylindrical preform, $26 \mathrm{~mm}$ in diameter and $25 \mathrm{~cm}$ long, which consisted of the GAST glass core contacted by four metallic alloy conduits that were encapsulated in a protective polymer cladding. The preform was consolidated for $60 \mathrm{~min}$ at $230^{\circ} \mathrm{C}$ under vacuum $\left(10^{-3}\right.$ Torr $\left.=0.133 \mathrm{~Pa}\right)$ in a three-zone horizontal tube furnace while rotating the preform around its axis. Subsequently, the preform was drawn in a three-zone vertical tube furnace with the top-zone temperature between 165 and $200^{\circ} \mathrm{C}$, and the middle-zone temperature $270^{\circ} \mathrm{C}$.

Array Data Acquisition and Image Processing: The metal electrodes in each fiber were connected to an electrical circuit. Each fiber device was placed in series with a $50 \mathrm{~V}$ dc power supply and a $20 \mathrm{k} \Omega$ resistor. 
The voltage drop on the resistor was monitored with an analog-to-digital card (National Instruments, DAQCard-6062E). The data from the rows and columns were arranged in two vectors, and the two-dimensional outer product of these one-dimensional vectors was calculated, producing an $8 \times 8$ matrix. In this way a localized excitation, such as the touch of a finger, was easily identified.

Received: October 4, 2005 Final version: December 7, 2005

[1] G. Busse, D. Wu, W. Karpen, J. Appl. Phys. 1992, 71, 3962

[2] V. P. Jackson, R. E. Hendrick, S. A. Feig, D. B. Kopans, Radiology 1993, 188, 297.

[3] V. J. Lumelsky, M. S. Shur, S. Wagner, IEEE Sens. J. 2001, 1, 41.
[4] T. Someya, T. Sekitani, S. Iba, Y. Kato, H. Kawaguchi, T. Sakurai, Proc. Natl. Acad. Sci. USA 2004, 101, 9966.

[5] K. T. V. Grattan, T. Sun, Sens. Actuators A 2000, 82, 40.

[6] M. Bayindir, F. Sorin, A. F. Abouraddy, J. Viens, S. D. Hart, J. D. Joannopoulos, Y. Fink, Nature 2004, 431, 826.

[7] M. Bayindir, O. Shapira, D. Saygin-Hinczewski, J. Viens, A. F. Abouraddy, J. D. Joannopoulos, Y. Fink, Nat. Mater. 2005, 4, 820.

[8] V. Q. Nguyen, J. S. Sanghera, F. H. Kung, P. C. Pureza, I. D. Aggarwal, J. Lightwave Technol. 2000, 18, 1395.

[9] N. F. Mott, Philos. Mag. 1969, 19, 835.

[10] N. F. Mott, E. A. Davis, Electronic Processes in Non-Crystalline Materials, Oxford University Press, New York 1979.

[11] M. A. Popescu, Non-Crystalline Chalcogenides, Kluwer, Dordrecht, The Netherlands 2000, p. 115.

[12] V. K. Tikhomirov, D. Furniss, A. B. Seddon, J. A. Savage, P. D. Mason, D. A. Orchard, K. L. Lewis, Infrared Phys. Technol. 2004, 45, 115 . 